\title{
DIRICHLETSCHE ABBILDUNGEN
}

\author{
C. CONSTANTINESCU
}

1. Für jede Riemannsche Fläche $R$ bezeichnen wir mit $M(R)$ die Algebra von Royden auf $R$ und mit $R^{*}$ (bzw. $\Delta_{R}$ )- die Kompaktifizierung (bzw. den idealen Rand) von Royden von $R$ ([5]). Für jede Funktion $\xi \in M(R)$ sei $J_{\xi}$ das kompakte Segment $\left[\inf _{R} \xi, \sup _{R} \xi\right]$ und $X(R)$ der kompakte Raum $\prod_{\xi \in M(R)} J_{\xi}$. Laut der Definition von $R^{*}$ ist jede Funktion $\xi \in M(R)$ auf $R^{*}$ (in eindeutiger Weise) stetig fortsetzbar. Bezeichnet man ebenfalls mit $\xi$ die so fortgesetzte Funktion und mit $\varphi_{R}$ die Abbildung $q \rightarrow(\xi(q))_{\xi \in M(R)}$ von $R^{*} \rightarrow X(R)$, so ist $\varphi_{R}$ ein Homeomorphismus von $R^{*}$ auf $\varphi_{R}\left(R^{*}\right)$.

Es seien $R, R^{\prime}$ zwei Riemannsche Flächen und $f$ eine nicht konstante analytische Abbildung von $R$ in $R^{\prime}$. Wir sagen, dass $f$ eine Dirichletsche $A b$ bildung ist, falls für jede Funktion $\xi^{\prime} \in M\left(R^{\prime}\right)$, die Funktion $\xi^{\prime} \circ f$ auf $R^{*}$ stetig fortsetzbar ist. In diesem Falle ist auch $f$ auf $R^{*} \rightarrow R^{\prime *}$ (in eindeutiger Weise) stetig fortsetzbar. In der Tat, die Abbildung $q \rightarrow\left(\xi^{\prime} \circ f(q)\right)_{\xi \in M\left(R^{\prime}\right)}$ ist eine stetige Abbildung von $R^{*}$ in $X\left(R^{\prime}\right)$. Offenbar wird $R^{*}$ in $\varphi_{R^{\prime}}\left(R^{\prime *}\right)$ abgebildet. Wendet man noch die stetige Abbildung ${\stackrel{-1}{R^{\prime}}}^{-1} \varphi_{R^{\prime}}\left(R^{*}\right) \rightarrow R^{*}$ an, so erhält man die gesuchte Fortsetzung von $f$.

Es gibt zwei wichtige Fälle, wenn $f$ eine Dirichletsche Abbildung ist. $1^{\circ} f$ ist endlichblättrig, d.h. es gibt eine natürliche Zahl $n$, derart dass $f^{-1}\left(p^{\prime}\right)$ aus höchstens $n$ Punkten besteht, für jeden $p^{\prime} \in R^{\prime}$. Dann ist sogar $\xi^{\prime} \circ f \in M(R)$ für jede $\xi^{\prime} \in M\left(R^{\prime}\right) .2^{\circ} R^{\prime}$ ist die Riemannsche Zahlenkugel $|w| \leq \infty$, und $f$ hat ein endliches sphärisches Integral, d.h. es ist

$$
\iint_{R} \frac{\left|f^{\prime}(z)\right|^{2} d x d y}{\left(1+|f(z)|^{2}\right)^{2}}<\infty
$$

In der Tat, sei $D_{n}^{\prime}$ die Menge der Punkte $p^{\prime} \in R^{\prime}$, für die $f^{-1}\left(p^{\prime}\right)$ genau aus $n$ Punkten besteht, und $a_{n}$ der sphärische Flächeninhalt von $D_{n}^{\prime}$. Es ist

$$
\sum_{n=1}^{\infty} n a_{n}<\infty
$$

Received June 25, 1961. 
Hat $\xi^{\prime} \in M\left(R^{\prime}\right)$ stetige Ableitungen, so ist $\left|\operatorname{grad} \xi^{\prime}\right|$ beschränkt, wo der Gradient in bezug auf die Metrik der Sphäre genommen wurde. Es ist also $\left|\operatorname{grad} \xi^{\prime}\right| \leq a<\infty$,

$$
\iint_{R}\left|\operatorname{grad}\left(\xi^{\prime} \circ f\right)\right|^{2} d x d y \leq a^{2} \sum_{n=1}^{\infty} n a_{n}<\infty,
$$

und $\xi^{\prime} \circ f \in M(R)$. Die Funktionen aus $M\left(R^{\prime}\right)$ mit stetigen Ableitungen bilden eine dichte Teilmenge in $M\left(R^{\prime}\right)$ (in der gleichmässigeren Topologie). Somit ist $\xi^{\prime} \circ f$ auf $R^{*}$ stetig fortsetzbar, für jede Funktion $\xi^{\prime} \in M\left(R^{\prime}\right)$.

2. In diesem Abschnitt wollen wir zuerst einige Hilfssätze feststellen. Es sei $G$ eine offene Menge auf $R$ und $u \in H P(R)$. Wir bezeichnen mit $I_{G} u$ die obere Grenze der subharmonische Minoranten von $u$, die auf $R-G$ verschwinden; $I_{G} u$ ist auf $G$ harmonisch. Es sei $\left(R_{n}\right)_{n}$ eine normale Ausschöpfung von $R$, und $u_{n}$ die Lösung des Dirichletschen Problems auf $R_{n} \cap G$ mit Randwerten $u$ auf $\bar{G} \cap \operatorname{Fr} R_{n}$ und 0 auf $R_{n} \cap \operatorname{Fr} G$. Die Folge $\left(u_{n}\right)$ ist abnehmend und

$$
I_{G} u=\lim _{n \rightarrow \infty} u_{n}
$$

auf $G$.

Hilfssatz 1. Es sei $G$ eine offene Menge auf $R$ und $u \in H P(R)$. Es ist

$$
\liminf _{p \rightarrow s} \operatorname{Iu}_{G} u(p)=\liminf _{p \rightarrow s} u(p),
$$

für jeden Punkt $s \in \Delta_{R}-\overline{R-G}$.

Es sei $s \in \Delta_{R}-\overline{R-G}$. Man kann immer annehmen, indem man eventuell $G$ verkleinert, dass alle Randpunkte von $G$ für das Dirichletsche Problem regulär sind. Es gibt eine positive Funktion $\xi \in M(R)$, die die Funktion $u$ minoriert, die auf $R-G$ verschwindet und für die

$$
\xi(s)>\liminf _{p \rightarrow s} u(p)-\varepsilon \quad(\varepsilon>0)
$$

ist. Es sei $\left(R_{n}\right)$ eine normale Ausschöpfung von $\mathrm{R}$. Wir bezeichnen mit $\xi_{n}$ die auf $R^{*}$ stetige Funktion, welche auf $R^{*}-R_{n} \cap G$ gleich $\xi$ und auf $R_{n} \cap G$ harmonisch ist. Offenbar gehört $\xi_{n}$ der Menge $M(R)$ an, und $\xi-\xi_{n}$ hat einen kompakten Träger. Indem man zu einer Teilfolge übergeht. kann man annehmen, dass $\left(\xi_{n}\right)$ konvergiert; wir setzen 


$$
\eta=\lim _{n \rightarrow \infty} \xi_{n}
$$

Die Funktion $\eta$ ist auf $R-G$ gleich 0 und auf $G$ harmonisch. Es ist leicht $z \mathbf{u}$ ersehen, dass $\eta \in M(R)$ und $\left(\xi_{n}\right)$ gegen $\eta$ in der $B D$-Topologie ([5]) konvergiert. Daraus folgt, dass $\eta=\xi$ auf $\Delta_{R}$ ist. Da, offenbar, $I_{G} u \geq \eta$ auf $G$ ist, so ist auch

$$
\liminf _{p \rightarrow s} I_{G} u(p) \geq \eta(s)=\xi(s)>\liminf _{\nu \rightarrow s} u(p)-\varepsilon .
$$

Somit ist

$$
\liminf _{p \rightarrow s} I u(p) \geq \liminf _{p \rightarrow s} u(p) .
$$

Die umgekehrte Ungleichung folgt sofort aus

$$
I_{G} u \leq u \text {. }
$$

Hilfssatz 2. Es sei $R$ eine hyperbolische Riemannsche Fläche und $K$ eine kompakte Menge auf $R^{*}-\left(R \cup \Delta_{R}\right)$. Es gibt eine stetige endliche positive superharmonische Funktion $S$ auf $R$, für die

$$
\lim _{p \rightarrow K} S(p)=\infty
$$

ist.

Es sei $G$ eine offene Menge auf $R^{*}$, die $\Delta_{R}$ enthält, und deren Randpunkte regulär für das Dirichletsche Problem sind. Dann ist $I_{G} 1$ stetig und subharmonisch auf $R$. Die Funktion $u=1-I_{G} 1$ ist stetig superharmonisch und positiv auf $R$, gleich 1 auf $R-G$ und, laut des Hilfssatzes 1 , ist

$$
\lim _{p \rightarrow \Delta_{R}} u(p)=0
$$

Es sei jetzt $U$ eine offene Menge auf $R^{*}, K \subset U$, für die $R-\bar{U}$ nur reguläre Randpunkte für das Dirichletsche Problem hat, und derart, dass $\bar{U} \cap \Delta_{R}=\phi$ ist. Wir bezeichen mit $u_{n}$ die Funktion $1-I 1$, wo $G_{n}=(R-\bar{U}) \cup R_{n}$ ist. Offenbar ist die Folge $\left(u_{n}\right)$ nicht zunehmend. Sie ist somit konvergent. Ihre Grenzfunktion $v$ ist auf $R$ harmonisch und beschränkt und

$$
\lim _{p \rightarrow s} v(p)=0
$$

in jedem Punkt $s \in d_{R}$. Aus dem Maximumprinzip folgt dann

$$
v=0 \text {. }
$$


Man kann jetzt leicht eine zunehmende Folge von natürlichen Zahlen $\left(\boldsymbol{n}_{\boldsymbol{k}}\right)_{k}$ wählen, derart dass

$$
S=\sum_{k=1}^{\infty} u_{n_{k}}
$$

endlich und stetig auf $R$ ist. Die Funktion $S$ erfüllt offenbar alle Bedingungen des Hilfssatzes.

Hilfssatz 3. Es sei $G$ ein Gebiet auf $R$ und $S$ eine nach unten halbbeschränkte superharmonische Funktion auf $G$, für die

$$
\liminf _{p \rightarrow q} S(p) \geq a
$$

ist, in jedem Punkt $q \in F r G \cap\left(R \cup \Delta_{R}\right)$. Dann ist $S \geq a$.

Dieser Hilfssatz folgt auch aus [4] (Proposition 1) oder [5] (Lemma 2.1).

$\mathrm{Da}$ auf $G$ eine nach unten halbbeschränkte superharmonische Funktion existiert, ist $G$ eine hyperbolische Riemannsche Fläche. Es gibt somit eine kompakte Menge $K_{0}$ auf $R-G$, derart dass $R^{\prime}=R-K_{0}$ auch hyperbolisch ist. Es sei $\varepsilon$ eine positive Zahl, und $K$ die Menge der Punkte $g \in F r G$, für die

$$
\liminf _{p \rightarrow q} S(p) \leq a-\varepsilon
$$

ist. $K$ ist eine kompakte Menge, die laut der Bedingung des Hilfssatzes auf $R^{*}-\left(R \cup \Delta_{R}\right)$ liegt. $\quad$ Da $K_{0}$ eine kompakte Menge auf $R$ ist, so kann man die Mengen $R^{*}-\left(R \cup \Delta_{R}\right)-G_{0}, R^{\prime *}-\left(R^{\prime} \cup \Delta_{R^{\prime}}\right)-G_{0}$ identifizieren $\left(G_{0}\right.$ offen, $K_{0} \subset G_{0}$, $G_{0}$ relativ kompakt in $R$ ). Es gibt dann, nach dem Hilfssatz 2, eine endliche positive superharmonische Funktion $S_{0}$ auf $R^{\prime}$, für die

$$
\lim _{p \rightarrow K} S_{0}(p)=+\infty
$$

gilt. Für jedes $\varepsilon_{0}>0$ ist die Funktion $S+\varepsilon_{0} S_{0}$ auf $G$ superharmonisch und wir haben

$$
\liminf _{p \rightarrow F_{r} G}\left(S+\varepsilon_{0} S_{0}\right)(p) \geq a-\varepsilon .
$$

Somit ist

$$
S+\varepsilon_{0} S_{0} \geq a-\varepsilon .
$$

$\mathrm{Da} \varepsilon_{0}$ beliebig und $S_{0}$ endlich ist, so folgt daraus

$$
S \geq a-\varepsilon .
$$


$\varepsilon$ ist aber auch beliebig und deshalb haben wir

$$
S \geq a \text {. }
$$

3. Es sei $\mu$ das kanonische Mass auf $\Delta_{R}$ ([5]); wir nehmen an, dass $\mu$ ein Mass auf $R^{*}-R$ ist, mit $\mu\left(R^{*}-\left(R \cup \Delta_{R}\right)\right)=0$. Wir nennen eine Menge $A \subset R^{*}$ polar, falls $A-R$ von $\mu$ - Masse null ist und für jede Kreisscheibe $U$ auf $R$, die Menge $A \cap U$ von der (äusseren) Kapazität null ist. Für die Definition der polaren Menge kann $R$ beliebig vom hyperbolischen oder parabolischen Typ sein.

SATz 1. Ist $f: R \rightarrow R^{\prime}$ eine Dirichletsche Abbildung, und $A^{\prime}$ eine polare Menge auf $R^{*}$, so ist auch $f^{-1}\left(A^{\prime}\right)$ eine polare Menge auf $R^{*}$.

Es genügt zu zeigen, dass $\bar{f}^{-1}\left(A^{\prime}\right)-R$ vom $\mu$ - Masse null ist. Wir werden drei Fälle unterscheiden: a) $A^{\prime} \subset \Delta_{R^{\prime}}$, b) $A^{\prime} \subset R^{\prime}$, c) $A^{\prime} \subset R^{\prime *}-\left(R^{\prime} \cup \Delta_{R^{\prime}}\right)$.

a) Es sei $G^{\prime}$ eine offene Menge auf $\Delta_{R^{\prime}}$ und $K$ eine kompakte Menge, $K \subset f^{-1}\left(G^{\prime}\right)$; dann ist $f(K)$ eine kompakte Menge und $f(K) \subset G^{\prime}$. Wir nehmen eine Funktion $\xi^{\prime} \in H D\left(R^{\prime}\right)$, die auf $f^{\prime}(K)$ gleich 1 auf $\Delta_{R^{\prime}}-G^{\prime}$ gleich 0 ist, und für die $0 \leq \xi^{\prime} \leq 1$ auf $R^{\prime}$ gilt. Die Funktion $\xi^{\prime} \circ f$ ist dann gleich 1 auf $K$; daraus folgt

$$
\mu(K) \leq \xi^{\prime} \circ f\left(p_{0}\right) \leq \mu^{\prime}\left(G^{\prime}\right),
$$

wenn man die kanonische Masse $\mu$ und $\mu^{\prime}$ den Punkten $p_{0}$ und $f\left(p_{0}\right)$ assoziert ([5]). Es ist also

$$
\mu\left(f^{-1}\left(G^{\prime}\right)\right)=\sup \mu(K) \leq \mu^{\prime}\left(G^{\prime}\right)
$$

Ist $A^{\prime} \subset \Delta_{R^{\prime}}$, und $G^{\prime}$ eine offene Menge auf $\Delta_{R^{\prime}}$ mit $A^{\prime} \subset G^{\prime}$, so erhalten wir

$$
\mu\left(f^{-1}\left(A^{\prime}\right)\right) \leq \mu\left(f^{-1}\left(G^{\prime}\right) \cap \Delta_{R}\right) \leq \mu^{\prime}\left(G^{\prime}\right) .
$$

Es ist also

$$
\mu\left(f^{-1}\left(A^{\prime}\right)\right) \leq \inf \mu^{\prime}\left(G^{\prime}\right)=0 .
$$

b) Es sei jetzt $A^{r}$ eine Menge auf $R^{\prime}$, deren abgeschlossene Hülle in einer offenen Kreisscheibe $U^{\prime}$ enthalten ist, und deren äussere Kapazität verschwindet. Es sei $G^{\prime}$ eine offene Menge $A^{\prime} \subset G^{\prime} \subset \bar{G}^{\prime} \subset U^{\prime}$ und $\left(K_{n}^{\prime}\right)$ eine zunehmende Folge von kompakten Mengen, derart dass alle Randpunkte von $R^{\prime}-K_{n}^{\prime}$ für das Dirichletsche Problem regulär sind unả 


$$
\begin{aligned}
& K_{n}^{\prime} \subset \stackrel{\circ}{K}_{n+1}^{\prime}, \\
& \bigcup_{n=1}^{\infty} K_{n}^{\prime}=G^{\prime}
\end{aligned}
$$

ist. Die stetige Funktion $\xi_{n}^{\prime}$ auf $R^{\prime}$, die auf $R^{\prime}-U^{\prime}$ gleich 0 , auf $K_{n}^{\prime}$ gleich 1 und auf $U^{\prime}-K_{n}^{\prime}$ harmonisch ist, gehört der Menge $M\left(R^{\prime}\right)$ an. Deshalb ist $\xi_{n}^{\prime} \circ f$ auf $R^{*}$ stetig fortsetzbar. Sie ist auf $f^{-1}\left(U^{\prime}\right)$ superharmonisch und auf $f^{-1}\left(K_{n}^{\prime}\right) \cap \Delta_{R}$ gleich 1. Es sei $\omega_{n}$ das harmonische Mass von $f^{-1}\left(K_{n}^{\prime}\right)$. Dann ist

$$
S_{n}=\xi_{n}^{\prime} \circ f-\underset{f^{-1}\left(U^{\prime}\right)}{I} \omega_{n}
$$

auf $f^{-1}\left(U^{\prime}\right)$ superharmonisch und nach unten halbbeschränkt und es ist

$$
\underset{p \rightarrow q}{\liminf } S_{n}(p) \geq 0
$$

für jeden Punkt $q \in\left(R \cup \Delta_{R}\right) \cap F r f^{-1}\left(U^{\prime}\right)$. Laut des Hilfssatzes 3 ist $S_{n} \geq 0$. Daraus folgert man

$$
\underset{\substack{-1 \\ f\left(U^{\prime}\right)}}{I} \omega_{n} \leq \xi_{n}^{\prime} \circ f
$$

Ls sei $\omega_{G^{\prime}}$ das harmonische Mass von $f^{-1}\left(G^{\prime}\right) \cap \Delta_{R}$ und

$$
\xi_{G^{\prime}}^{\prime}=\lim _{n \rightarrow \infty} \xi_{n}^{\prime} .
$$

Aus obiger Ungleichung, $\omega_{n} \uparrow \omega_{G^{\prime}}$ und Satz 11 in [2] folgt

$$
\underset{-1}{I\left(U^{\prime}\right)} \underset{G^{\prime}}{I} \omega_{G^{\prime}}^{\prime} \circ \xi_{G^{\prime}}^{\prime} \circ f
$$

Es sei $\left(G_{n}^{\prime}\right)$ eine abnehmende Folge von offenen Mengen, die die Menge $A^{\prime}$ enthalten, mit $\bar{G}_{1}^{\prime} \subset U^{\prime}$ und deren Kapazität gegen 0 konvergiert. Dann ist

$$
\lim _{n \rightarrow \infty} \xi_{G^{\prime} n}^{\prime}\left(p^{\prime}\right)=0
$$

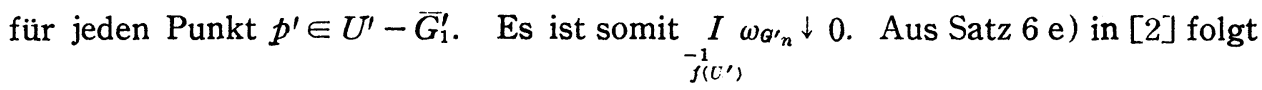
$\underset{-1}{E} \underset{-1}{I} \omega_{G^{\prime} n} \downarrow 0$. Laut des Hilfssatzes 1 ist ${ }^{-1}\left(C^{\prime}\right) \stackrel{-1}{f\left(U^{\prime}\right)}$

$$
\liminf _{y \rightarrow s} \underset{\substack{-1 \\ f\left(U^{\prime}\right)}}{E} \underset{\substack{-1 \\ f\left(U^{\prime}\right)}}{I} \omega_{G^{\prime} n}(p) \geq \liminf _{p \rightarrow s} \underset{\substack{-1 \\ f\left(U^{\prime}\right)}}{I} \omega_{G^{\prime} n}(p)=\liminf _{p \rightarrow s} \omega_{G^{\prime} n}(p)=1
$$

für jeden Punkt $s \in f^{-1}\left(G_{n}^{\prime}\right) \cap \Delta_{R}$. Daraus folgt 


$$
\underset{-1}{E} \underset{\substack{-1 \\ f^{\prime}\left(U^{\prime}\right)}}{E} \underset{f\left(U^{\prime}\right)}{I} \omega_{G^{\prime} n} \geq \omega_{G^{\prime} n}
$$

Die Menge $f^{-1}\left(A^{\prime}\right) \cap \Delta_{R}$ ist somit vom $\mu$-Masse null.

Ist $A^{\prime}$ eine beliebige Menge auf $R^{\prime}$, deren äussere Kapazität verschwindet, so kann man Mengen $A_{n}^{\prime}$ finden, die die obige Bedingung erfüllen und deren Vereinigung gleich $A^{\prime}$ ist. Es ist

$$
\bar{f}^{-1}\left(A^{\prime}\right) \cap \Delta_{R}=\bigcup_{n=1}^{\infty}\left(f^{-1}\left(A_{n}^{\prime}\right) \cap \Delta_{R}\right)
$$

$\operatorname{Da} f^{-1}\left(A_{n}^{\prime}\right) \cap \Delta_{R}$ vom $\mu$ - Masse null sind, so ist auch $f^{-1}\left(A^{\prime}\right) \cap \Delta_{R}$ vom $\mu$-Masse null.

c) Die Menge $f^{-1}\left(R^{\prime *}-\left(R^{\prime} \cup \Delta_{R^{\prime}}\right)\right)$ ist offen auf $\Delta_{R}$. Es sei $K$ eine kompakte Menge $K \subset f^{-1}\left(R^{\prime *}-\left(R^{\prime} \cup \Delta_{R^{\prime}}\right)\right)$; dann ist $f(K)$ kompakt, und $f(K) \subset R^{*}-\left(R^{\prime} \cup \Delta_{R^{\prime}}\right)$. Ist $R^{\prime}$ hyperbolisch, so gibt es, laut des Hilfssatzes 2 , eine endliche positive superharmonische Funktion $S^{\prime}$ auf $R^{\prime}$, für die

$$
\lim _{\nu^{\prime} \rightarrow J(K)} S^{\prime}\left(p^{\prime}\right)=\infty
$$

gilt. Die Funktion $S^{\prime} \circ f$ ist dann endlich, positiv und superharmonisch auf $R$, und wir haben

$$
\lim _{p \rightarrow K} S^{\prime} \circ f(p)=\infty
$$

Wir bezeichnen mit $\omega$ das harmonische Mass von $K$; es ist

$$
\begin{aligned}
& \omega\left(p_{0}\right)=\mu(K), \\
& \lim _{p \rightarrow \perp R-K} \omega(p)=0 .
\end{aligned}
$$

Die Funktion $S_{n}=S^{\prime} \circ f-n \omega$ ist auf $R$ nach unten halbbeschränkt und superharmonisch und es gilt

$$
\liminf _{p \rightarrow \Delta R} S_{n}(p) \geq 0
$$

Aus dem Hilfssatz 3 folgt

$$
S_{n} \geq 0
$$

Daraus ergibt sich 


$$
\begin{gathered}
n \omega \leq S^{\prime} \circ f, \\
\mu(K)=\omega\left(p_{0}\right) \leq \frac{1}{n} S^{\prime}\left(p_{0}^{\prime}\right), \\
\mu(K)=0 .
\end{gathered}
$$

Es ist somit

$$
\mu\left(f^{-1}\left(R^{\prime *}-\left(R^{\prime} \cup \Delta_{R^{\prime}}\right)\right)\right)=\sup \mu(K)=0
$$

Ist $R^{\prime}$ parabolisch, so ist $f^{-1}\left(R^{\prime *}-R^{\prime}\right)$ vom $\mu$ - Masse null. Das kann ebenso bewiesen werden, wie wir oben gezeigt haben, dass $f^{-1}\left(A^{\prime}\right) \cap \Delta_{R}$ vom $\mu$-Masse null ist, wenn $A^{\prime} \subset R^{\prime}$ und von äusserer Kapazität null ist. Damit ist der Satz vollständig bewiesen.

Wir bezeichnen mit $U_{H D}([1])$ die Klasse der Riemannschen Flächen, die eine $H D$ (oder $\underline{H D}$ )-minimale Funktion besitzen. Diese ist, wie Nakai gezeigt hat ([5]), gerade die Klasse der Riemannschen Flächen $R$, die einen Punkt $s \in \Delta_{R}$ besitzen, dessen $\mu$-Mass positiv ist.

Folgesatz 1. Ist $f: R \rightarrow R^{\prime}$ eine Dirichletsche Abbildung und $R \in U_{H D}$, so ist auch $R^{\prime} \in U_{H D}$.

Es sei $s$ ein Punkt auf $\Delta_{R}$, dessen $\mu$-Mass positiv ist. Die Menge $\{f(s)\}$ kann nicht polar sein. Daraus folgt sofort, dass $f(s)$ der Menge $\Delta_{R^{\prime}}$ angehört und $\{f(s)\}$ ein positives $\mu^{\prime}$-Mass hat. Es ist also $R^{\prime} \in U_{H D}$.

Folgesatz 2. Jede Fortsetzung einer Riemannschen Fläche aus der Klasse $U_{H D}$ gehört auch der Klasse $U_{H D}$ an.

Dieser Satz wurde in [1] (Satz 11) mittels der universellen Überlagerungsfläche bewiesen.

Folgesatz 3. Es ist

$$
U_{H D} \subset O_{A D}-O_{G}
$$

Jede $A D$-Funktion $f$ ist nähmlich eine Dirichletsche Abbildung.

Auch dieser Satz wurde in [1] (Satz 7) mittels der universellen Überlagerungsfläche bewiesen.

Folgesatz 4. Es sei $R$ ein Gebiet auf der Riemannschen Kugel $|z| \leq \infty$. Der relative Rand von $R$ ist ein Quotientenraum des kompakten Raumes $R^{*}-R$. 
Die identische Abbildung ist eine Dirichletsche Abbildung.

4. Satz 2. Es sei $R$ ein hyperbolisches Gebiet auf der Riemannschen Kugel, $\psi$ eine stetige Funktion auf dem relativen Rand von $R$ und $H_{R}^{\psi}$ die Lösung des Dirichletsihen Problems auf $R$ mit $\psi$ als Randfunktion. Es sei $z_{0}$ ein Punkt des relativen Randes von $R$ und $\left(z_{n}\right)$ eine Punktfolge aus $R$, die gegen $z_{0}$ konvergiert und die auf $R^{*}$ gegen $A_{R}$ konvergiert. Dann ist

$$
\lim _{n \rightarrow \infty} H_{\dot{R}}^{\psi}\left(z_{n}\right)=\psi\left(z_{0}\right)
$$

Es sei $z_{1} \in R$ und $g_{z_{1}}$ die Greensche Funktion von $R$ mit $z_{1}$ als Pol. Die Funktion $h=\min \left(g_{z_{1}}, 1\right)$ gehört $z u(R)$. Da sie in der $B D$-Topologie die Grenzfunktion einer Folge von Funktionen aus $M(R)$ mit kompakten Trägern ist, so ist

$$
\lim _{n \rightarrow \infty} h\left(z_{n}\right)=0 .
$$

Die Behauptung des Satzes stimmt offenbar, falls $z_{0}$ für das Dirichletsche Problem regulär ist. Es genügt also den Satz unter der Voraussetzung zu beweisen, dass $z_{0}$ nicht regulär ist. Es gibt dann eine Folge von einfach zusammenhängenden Gebieten $\left(G_{m}\right)$, deren Rand in $R$ liegt, und für die

$$
G_{m} \supset \bar{G}_{m+1}, \quad \bigcap_{m=1}^{\infty} G_{m}=\left\{z_{0}\right\}
$$

ist. Wir bezeichnen mit $S_{m}$ die Funktion auf $R$, die auf $R-G_{m}$ gleich 1 und auf $R \cap G_{m}$ gleich der Lösung des Dirichletschen Problems ist, mit Randwerten 1 auf dem Rande von $G_{m}$ und 0 auf dem Rande von $R$, der in $G_{m}$ liegt. Offenbar ist $S_{m}$ superharmonisch in $R$, und es gibt eine reele $Z$ ahl $a_{m}$, derart dass

$$
S_{m} \leq a_{m} h
$$

auf $G_{m} \cap R$ ist. Daraus folgt

$$
\lim _{n \rightarrow \infty} S_{m}\left(z_{n}\right)=0
$$

Es sei

$$
S=\sum_{m=1}^{\infty} \frac{1}{2^{m}} S_{m}
$$

$S$ ist eine positive superharmonische Funktion auf $R$, für die

$$
\liminf _{z \rightarrow \zeta} S(z)>0
$$


ist, für jeden Randpunkt $\zeta$ von $R$, der von $z_{0}$ verschieden ist. Ausserdem haben wir

$$
\limsup _{n \rightarrow \infty} S\left(z_{n}\right) \leq \frac{1}{2^{k}}+\sum_{m=1}^{k} \frac{1}{2^{m}} \limsup _{n \rightarrow \infty} S_{m}\left(z_{n}\right) \leq \frac{1}{2^{k}}
$$

und somit

$$
\lim _{n \rightarrow \infty} S\left(z_{n}\right)=0
$$

Der Beweis verläuft jetzt weiter auf dem gewöhnlichen Wege.

5. Es sei $f$ eine beliebige analytische Abbildung von $R$ in $R^{\prime}$ und $G^{\prime}$ die Menge der Punkte $p^{\prime} \in R^{\prime}$ die eine Umgebung $U^{\prime}$ besitzen, so dass die Überlagerung von $U^{\prime}$ durch $f$ einen endlichen Flächeninhalt hat, d.h. es ist

$$
\iint_{\substack{-1 \\ f\left(U^{\prime}\right)}} \frac{d f^{2}}{d \bar{z}} d x d y<\infty,
$$

wenn $U^{\prime}$ mit der Kreisscheibe $|w|<1$ indentifiziert wird. Die Komplementärmenge $F^{\prime}$ von $G^{\prime}$ ist offenbar abgeschlossen.

SATz 3. Ist $R^{\prime}$ kompakt und enthält $F^{\prime}$ kein Kontinuum, so ist $f$ eine Dirichletsche Abbildung.

Es sei $\xi^{\prime}$ eine Funktion mit stetiger Ableitung auf $R^{\prime}$ und $\varepsilon>0$. Man kann endlich viele einfach zusammenhängende paarweise punktfremde Gebiete $G_{1}^{\prime}, \ldots, G_{n}^{\prime}$ finden, derart dass der Rand von $G_{n}^{\prime}$ analytisch und in $G^{\prime}$ enthalten ist, die Schwankung von $\xi^{\prime}$ auf $G_{i}^{\prime}(i=1, \ldots, n)$ kleiner als $\varepsilon$ und

$$
F^{\prime} \subset \bigcup_{i=1}^{n} G_{i}^{\prime}
$$

ist. Es seien $H_{1}^{\prime}, \ldots, H_{n}^{\prime}$ einfach zusammenhängende Gebiete mit analytischem Rande, derart dass

$$
\bar{H}_{i}^{\prime} \subset G_{i}^{\prime}, \quad \bar{G}_{i}^{\prime}-H_{i}^{\prime} \subset G^{\prime}
$$

ist, und $\xi_{0}^{\prime}$ sei diejenige stetige Funktion auf $R^{\prime}$, die auf $R^{\prime}-\bigcup_{i=1}^{n} G_{i}^{\prime}$ glich $\xi^{\prime}$, auf $\bar{H}_{i}^{\prime}$ gleich $\xi^{\prime}\left(p_{i}^{\prime}\right)$, wo $p_{i}^{\prime} \in G_{i}^{\prime}$, und auf $G_{i}^{\prime}-\bar{H}_{i}^{\prime}$ harmonisch ist. Da $\hat{s}_{0}^{\prime}$ auf jedem $H_{i}^{\prime}$ konstant ist, so folgt sofort, dass $\hat{5}_{0}^{\prime} \circ f \in M(R)$ und somit auf $R^{*}$ stetig fortsetzbar ist. Da $\left|\xi^{\prime}-\xi_{0}^{\prime}\right|<\varepsilon$, und $\varepsilon$ beliebig war, so ergibt sich, dass auch $\xi^{\prime} \circ f$ auf $R^{*}$ stetig fortsetzbar ist. Da die Funktionen mit stetiger Ableitung in 
der gleichmässigen Topologie von $M\left(R^{\prime}\right)$ dicht sind, so ist für jede Funktion $\xi^{\prime}$ aus $M\left(R^{\prime}\right), \xi^{\prime} \circ f$ auf $R$ stetig fortsetzbar und somit ist $f$ eine Dirichletsche Abbildung.

Die Umkehrung dieses Satzes stimmt nicht. Wir werden zwar ein Beispiel einer Dirichletschen Abbildung $f$ angeben in welchem $R^{\prime}$ die Riemannsche Kugel und $F^{\prime}$ das Quadrat $|x|<1,|y|<1$ ist. Wir bilden die Riemannsche Fläche $R$ auf folgende Art. Die erste "Schichte" soll aus einem Blatt $|x|<1,|y|<1$ bestehen. Die zweite wird aus vier Blättern bestehen und zwar $0<x<1$, $0<y<1 ; \quad-1<x<0, \quad 0<y<1 ; \quad-1<x<0, \quad-1<y<0 ; \quad 0<x<1, \quad-1<y<0$. Jedes Blatt dieser Schichte soll mit dem Blatt der ersten Schichte mittels eines Einschnittes kreuzweise verknüpft werden. Die dritte Schichte besteht aus 16 Blättern, die durch Einteilung jedes Blattes der zweiten Schichte in vier Quadrate entstehen; diese werden kreuzweise (mittels eires Einschnittes) mit den Blättern der zweiten Schichte verknüpft. Setzt man dieses Verfahren fort, so erhält man eine Riemannsche Fläche $R$. Die Abbildung $f$ soll die Projektion von $R$ auf $R^{\prime}$ sein. Es ist leicht zu erkennen, dass $f$ die obenerwähnten Eigenschaften besitzt. Durch eine kleine Veränderung der Konstruktion kann man sogar ein Beispiel bilden, in welchem $F^{\prime}$ mit $R^{\prime}$ zusammenfällt.

6. SAtz 4. Jede Dirichletsche Abbildung ist eine Fatousche Abbildung ([2]).

Es sei $f: R \rightarrow R^{\prime}$ eine Dirichletsche Abbildung und $U^{\prime}$ eine Kreischeibe auf $R^{\prime}$ die wir mit $|z|<1$ identifizieren. Es sei $\Theta_{r}^{\prime}$ der Kreis $|z|=r$. Da nur für abzähibar viele $r, f^{-1}\left(\Im_{r}^{\prime}\right) \cap \Delta_{R}$ ein positives $\mu$-Mass hat, so gibt es ein $r$, derart dass $f^{-1}\left(\Im_{r}^{\prime}\right) \cap \Delta_{R}$ ein verschwindendes $\mu$-Mass hat. Bezeichnet man mit $G$ die offene Menge $f^{-1}\left(R^{\prime}-\Xi_{r}^{\prime}\right) \cap R$, so ist

$$
\overline{R-G} \subset f^{-1}\left(\varsigma_{r}^{\prime}\right)
$$

Aus dem Hilfssatz 1 folgt, dass

$$
\liminf _{i \rightarrow s} \underset{G i}{E} I 1(p) \geq \liminf _{p \rightarrow s} I_{G} 1(p)=1
$$

ist, für jeden Punkt $s \in \Delta_{R}-f^{-1}\left(\Xi_{r}^{\prime}\right)$. Aus dem Verallgemeinerten Minimumsatz in [5] (Theorem 2.4) folgert man

$$
\underset{G}{E} I_{G} 1=1
$$


was gerade aussagt, dass $f$ eine Fatousche Abbildung ist.

HiLfsSATz 4. Es sei $G$ eine offene Menge auf $R$, deren Randpunkte in bezug auf $R$ für das Dirichletsche Problem regulär sind und $\xi$ eine stetige Funktion auf $R^{*}$, die auf $R-G$ verschwindet. Dann gibt es eine Funktion $u$, die auf $R^{*}$ stetig, auf $G$ harmonisch, auf $R-G$ null und auf $\Delta_{R}$ gleich $\xi$ ist.

Wir nehmen zuerst an, dass $\xi \in M(R)$. Es sei $\left(R_{n}\right)$ eine normale Ausschöpfung von $R$ und $v_{n}$ die auf $R^{*}$ stetige Funktion, die auf $R-R_{n} \cap G$ gleich $\xi$ und auf $R_{n} \cap G$ harmonisch ist. Die Funktion, $\xi-v_{n}$ gehört zu $M(R)$ und hat einen kompakten Träger. Indem man zu einer Teilfolge übergeht, kann man annehmen, dass $\left(v_{n}\right)$ konvergiert und es sei $v$ ihre Grenzfunktion. Man sieht leicht, dass $\xi-v_{n}$ gegen $\xi-v$ in $\operatorname{der} B D$-Topologie konvergiert, so dass $\xi-v$ auf $A_{R}$ verschwindet.

In dem allgemeinen Fall gibt es eine Folge $\left(\xi_{n}\right)$ von Funktionen aus $M(R)$, die gleichmässig gegen $\xi$ konvergieren. Laut obigen Betrachtungen gibt es eine Folge $\left(v_{n}\right)$ von stetigen Funktionen auf $R^{*}$, die auf $\Delta_{R}$ und $R-G$ gleich $\xi_{n}$ und auf $G$ harmonisch sind. Mittels des Hilfssatzes 3 sieht man, dass $v_{n}$ gleichmässig gegen eine Funktion $u$ konvergiert. Man überzeugt sich sofort, dass $u$ die Bedingungen des Hilfssatzes erfüllt.

SaTz 5. Eine Dirichletsche Abbildung $f: R \rightarrow R^{\prime}$ ist vom Typus $B l$ ([3]) dann und nur dann, wenn

$$
f\left(\Delta_{R}\right) \cap R^{\prime}=\varnothing
$$

ist. Diese Beziehung ist zu

$$
f\left(\Delta_{R}\right) \subset \Delta_{R^{\prime}}
$$

äquivalent.

Es sei

$$
f\left(\Delta_{R}\right) \cap R^{\prime} \neq \phi .
$$

Dann gibt es eine kompakte Menge $K$ auf $\Delta_{R}$, die ein positives $\mu$-Mass hat und für die

$$
f(K) \subset R^{\prime}
$$

ist. Wir nehmen eine offene Menge $G^{\prime} \subset R^{\prime}$, die die Menge $f(K)$ enthält und einen analytischen Rand hat, und eine Funktion $\xi^{\prime} \in M\left(R^{\prime}\right), 0 \leq \xi^{\prime} \leq 1$, die auf $R^{\prime}-G^{\prime}$ 
gleich 0, auf $f(K)$ gleich 1 und auf $G^{\prime}$ superharmonisch ist. Dann ist $\xi^{\prime} \circ f$ stetig auf $R^{*}$ und verschwindet auf $R-f^{-1}\left(G^{\prime}\right)$. Laut des vorangehenden Hilfssatzes, gibt es eine stetige Funktion $u$ auf $R^{*}$, die auf $R-f^{-1}\left(G^{\prime}\right)$ verschwindet, auf $f^{-1}\left(G^{\prime}\right)$ harmonisch und auf $\Delta_{R}$ gleich $\xi^{\prime} \circ f$ ist. Die Funktion $\xi^{\prime} \circ f-u$ ist auf $f^{-1}\left(G^{\prime}\right)$ stetig, superharmonisch, beschränkt und es ist

$$
\liminf _{p \rightarrow q}\left(\xi^{\prime} \circ f(p)-u(p)\right) \geq 0,
$$

für jeden Punkt $q \in \operatorname{Fr}^{-1}\left(G^{\prime}\right) \cap\left(R \cup \Delta_{R}\right)$. Laut des Hilfssatzes 3 ist

$$
\dot{\xi}^{\prime} \circ f \geq u \text {. }
$$

Es ist also $u \leq 1$ und, da $u$ auf dem relativen Rande von $f^{-1}\left(G^{\prime}\right)$ verschwindet,

$$
u \leq \underset{\substack{-1 \\ f\left(G^{\prime}\right)}}{I} 1
$$

Auf $K$ ist $u$ gleich 1. Desto mehr ist $\underset{\substack{-1 \\ f\left(G^{\prime}\right)}}{E} u$ gleich 1 auf $K$ und deshalb haben wir

$$
0<\mu(K) \leq \underset{\substack{-1 \\ f\left(a^{\prime}\right)}}{E} u\left(p_{i j}\right)
$$

Es ist also

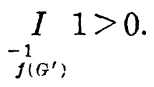

Aus Satz 27 in [2] folgt, dass $f$ keine Abbildung vom Typus $B l$ ist, denn (mit den Bezeichnungen von [2]) $\hat{f}$ ist fast überall auf $\Delta_{1}$ definiert (Satz 4 und [2] Satz 24) und fast überall auf $\Delta_{1}\left(f^{-1}\left(G^{\prime}\right)\right)$ wird $\hat{f}(s)$ in $\overline{G^{\prime}} \subset R^{\prime}$ enthalten sein.

Es sei jetzt

$$
f\left(A_{R}\right) \cap R^{\prime}=\phi .
$$

Die Menge $\Delta_{R}-f^{-1}\left(\Delta_{R^{\prime}}\right)$ ist offen auf $\Delta_{R}$. Aus dem Satz 1 folgt, dass sie vom $\mu$-Masse null ist. Sie ist also ([5] Proposition 2.1) leer und es ist $f\left(\Delta_{R}\right) \subset \Delta_{R^{\prime}}$ und $R^{\prime} \notin O_{G}$. Es sei $\left(K_{n}^{\prime}\right)$ eine normale Ausschöpfung von $R^{\prime}$. Es sei $\omega_{n}^{\prime} \in M\left(R^{\prime}\right)$, $\omega_{n}^{\prime}$ gleich 0 auf $\bar{R}_{n}^{\prime}$, gleich 1 auf $\Delta_{R^{\prime}}$ und harmonisch $R^{\prime}-\bar{R}_{n}^{\prime}$. $\omega_{n}^{\prime} \circ f$ ist gleich 1 überall auf $A_{R}$. Die Funktion $\omega_{n}^{\prime} \circ f$ ist auf $f^{-1}\left(R^{\prime}-\bar{R}_{n}^{\prime}\right)$ harmonisch, verschwindet auf dem relativen Rand und es ist

$$
0 \leq \omega)_{n}^{\prime} \circ f \leq 1
$$

Daraus folgt 


$$
\omega_{n}^{\prime} \circ f \leq \underset{-1}{f\left(R^{\prime}-\bar{R}^{\prime} n\right)} \underset{1}{I}
$$

Die Funktion $\underset{\substack{-1 \\ J\left(R^{\prime}-\bar{R}^{\prime} n\right)}}{E} \underset{\substack{-1 \\ f\left(R^{\prime}-\bar{R}^{\prime} n\right)}}{I} 1$ ist somit auf $\Delta_{R}$ gleich 1 ; wir haben also

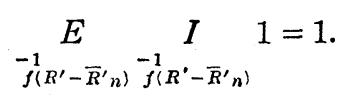

Daraus folgert man, dass für fast alle $s \in \Delta_{1}, \hat{f}(s) \notin R_{n}^{\prime}$ : Da $n$ beliebig ist, so folgt $\hat{f}(s) \in \Delta_{1}^{\prime}$ für fast alle $s \in \Delta_{1}$ und ([2] Satz 27) $f$ ist vom Typus $B l$.

Folgesatz 5. Ist $f: R \rightarrow R^{\prime}$ eine Dirichletsche Abbildung und $R \in O_{H \nu_{\infty}}$, so ist $f$ vom Typus $B l$ und $R^{\prime} \in O_{H D_{\infty}}$. Ist $R \in O_{I I n_{n}}-\bigcup_{i<n} O_{H D_{i}}$, so ist $R^{\prime} \in O_{H I D_{n^{\prime}}}-\bigcup_{i<n^{\prime}} O_{H D_{i}}$ für ein $n^{\prime}, n^{\prime} \leq n$.

Es sei $A$ die Menge der Pukte $s \in \Delta_{R}$, die ein positives $\mu$-Mass haben. $\quad D a$ $R \in O_{H D_{\infty}}$, so ist $\Delta_{R}-A$ vom $\mu$-Masse null, und deshalb ist $\Delta_{R}=\bar{A}$ ([5] Proposition 2.1). Aus dem Satz 1 folgt, dass

$$
f(A) \cap R^{\prime}=\phi
$$

ist. Es ist somit

$$
f\left(\Delta_{R}\right) \cap R^{\prime}=\varnothing
$$

und $f$ ist vom Typus $B l$.

Aus dem Satz 1 folgt weiter, dass jeder Punkt von $f(A)$ ein positives $\mu^{\prime}$-Mass hat. $\mathrm{Um} z \mathrm{u}$ beweisen, dass $R^{\prime}$ in $O_{H D_{\infty}}$ enthalten ist, bleibt nur zu zeigen, dass $\Delta_{R^{\prime}}-f(A)$ vom $\mu^{\prime}$-Masse null ist. Im entgegengesetzten Falle kann man eine Funktion $u^{\prime} \in H\left(R^{\prime}\right)$ finden, die

$$
\lim _{y \rightarrow f(A)} u^{\prime}\left(p^{\prime}\right)=1
$$

ist und für die

$$
0<\boldsymbol{u}^{\prime}<1
$$

auf $R^{\prime}$ gilt. Die Funktion $u^{\prime} \circ f$ ist auf $R$ harmonisch und beschränkt und es ist $\lim _{\nu \rightarrow 4} u^{\prime} \circ f(p)=1$. Aus dem verallgemeinerten Minimumprinzips ([5] Theorem 2.4) folgt aber die widersprechende Beziehung

$$
u^{\prime} \circ f=1 \text {. }
$$

Gehört die Riemannsche Fläche $R$ der Klasse $O_{H D_{n}}-\bigcup_{i<n} O_{H D_{i}}, n<\infty$, so enthält sie $n$ Punkte $s_{1}, \ldots, s_{n}$, die positives $\mu$-Mass haben. Es ist leicht zu sehen, 
dass das harmonische Mass von $\left\{s_{i}\right\}$ eine minimale $H D$-Funktion ist ([5]). Daraus folgt, wie Nakai gezeigt hat $([\bar{j}])$, dass $s_{i}$ isoliert in $\Delta_{R}$ liegt. Die Menge $\left\{s_{1}, \ldots, s_{n}\right\}$ ist somit abgeschlossen. $\mathrm{Da} \Delta_{R}-\left\{s_{1}, \ldots, s_{n}\right\}$ vom nullen $\mu$-Masse ist, ist sie leer ([5]). Die Menge $A_{R}$ besteht also genau aus den $n$ Punkten $s_{1}$, $\ldots, s_{n}$. Laut des Satzes 1 ist $\left\{f\left(s_{i}\right)\right\}$ vom positiven $\mu^{\prime}$-Masse. Es ist ausserdem $\Delta_{R^{\prime}}-\bigcup_{i=1}^{n}\left\{f\left(s_{i}\right)\right\}$ vom $\mu^{\prime}$-Masse null. Daraus folgt $R^{\prime} \in O_{H D_{n^{\prime}}}-\bigcup_{i<n^{\prime}} O_{H D_{i}}$ mit $n^{\prime} \leq n$.

\section{LITERATUR}

[1] Constantinescu, C. und Cornea, A.: Über den idealen Rand und einige seiner Anwendungen bei der Klassifikation der Riemannschen Flächen, Nagoya Math. Journ., 13 (1958), 169-233.

[2] Constantinescu, C. und Cornea, A.: Über das Verhalten der analytischen Abbildungen Riemannscher Flächen auf dem idealen Rand von Martin, Nagoya Math. Journ., 17 (1960), 1-87.

[3] Heins, M.: On the Lindelof principle, Ann. of Math., 61 (1955), 440-473.

[4] Kusunoki, Y. and Mori, S.: On the harmonic boundary of an open Riemann surface, Japan. Journ. Math., 29 (1959), 52-56.

[5] Nakai, M.: A measure on the harmonic boundary of a Riemann surface, Nagoya Math. Journ., 17 (1960), 181-218.

\section{Mathematisches Institut}

der Rumänischen Akademie 